\title{
PERCEPCIONES DE LA FUNCIÓN ORIENTADORA EN EDUCACIÓN SEXUAL DESDE LA PERSPECTIVA DEL TUTOR Y EL ALUMNO EN UNA INSTITUCIÓN EDUCATIVA
}

\author{
PERCEPTIONS OF THE GUIDING ROLE IN SEXUAL EDUCATION FROM THE PERSPECTIVE OF THE \\ GUARDIAN AND THE STUDENT IN AN EDUCATIONAL INSTITUTION
}

\author{
Gianinna Julca-Meneses (iD 1,a, Yasmine Karina Sotomayor-Torres (iD) 2,3,a,b
}

\begin{abstract}
Objetivo: Valorar las percepciones de la función orientadora en educación sexual desde la perspectiva del tutor y el alumno. Material y métodos: Estudio de enfoque cualitativo y diseño fenomenológico; se empleó la entrevista a profundidad y como instrumento de recolección de datos la guía de entrevista. Para determinar las muestras se tomaron en cuenta los criterios de selección, siguiendo el criterio de "saturación", con lo cual, finalmente se trabajó con dos muestras de estudio: 7 tutores y 10 alumnos. Los datos recogidos fueron organizados y analizados mediante el modelo de la Teoría fundamentada. Resultados: Se evaluaron las percepciones de la función orientadora en sus dimensiones: educación sexual (recursos disponibles, necesidades, fortalezas y debilidades), temas trascendentales de la educación sexual (plan de vida, autoestima, enamoramiento, inicio de relaciones sexuales, embarazo adolescente e infecciones de transmisión sexual), temas controversiales de la educación sexual (aborto, uso de métodos anticonceptivos y homosexualidad) y otros agentes involucrados (otros agentes dentro del colegio, familia-padres y personal de salud). Las percepciones de tutores y alumnos respecto a la función orientadora de este último en temas de educación sexual son discrepantes, hallándose como debilidades fundamentales la falta de capacitación de los tutores. Conclusión: Se requiere de una Educación Sexual Integral con enfoque de género que aborde adecuadamente todos los temas relacionados, pero que, además, incluya a otros agentes formadores como los padres de familia y personal de salud capacitado en salud sexual y reproductiva.
\end{abstract}

Palabras clave: Educación sexual, Estudiantes, Mentores (Fuente: DeCS, BIREME)

Objective: To assess the perceptions of the guiding function in sexual education from the perspective of the tutor and the student. Material and methods: Qualitative approach study and phenomenological design; the in-depth interview was used and as an instrument of data collection the interview guide. To determine the samples, the selection criteria were considered, following the criterion of "saturation", with which, finally, two study samples were worked on: 7 tutors and 10 students. The collected data were organized and analyzed using the Grounded Theory model. Results: The perceptions of the orienting function in its dimensions were evaluated: sexual education (resources available, needs, strengths and weaknesses), transcendental topics of sexual education (life plan, selfesteem, falling in love, initiation of sexual relations, adolescent pregnancy and sexually transmitted infections), controversial issues of sex education (abortion, use of contraceptive methods and homosexuality) and other agents involved (other agents within the school, family-parents and health personnel). The perceptions of tutors and students regarding the orientation function of the latter in sexual education issues are discrepant, being found as fundamental weaknesses the lack of training of tutors. Conclusion: The tutors perceive their guiding role in sex education as an important responsibility towards the students, which depends on the commitment and attitude of each tutor, weakened by the limited time and lack of information and training on sexual education issues. The students perceive the guiding function as inadequate and insufficient.

Key words: Sex education, Students, Mentors (Source: MeSH NLM)

Filiación:

1. Hospital de Emergencias de Villa El Salvador. Lima, Perú

2. Federal University of Vales do Jequitinhonha e Mecuri. Minas Gerais, Brasil.

3. WAREM S.A.C

a. Licenciada en Obstetricia.

b. Magíster en Salud, Sociedad y Ambiente.
Citar como: Julca-Meneses G

Sotomayor-Torres YK. Percepciones de la función orientadora en educación sexual desde la perspectiva del tutor y el alumno en una institución educativa. Revista Internacional de Salud Materno Fetal. 2020; 5 (3): 19-27. DOI:

https://doi.org/10.47784/rismf.2020.5.3.90
Financiamiento: Autofinanciado Conflictos de interés: Las autoras declaran no presentar conflictos de interés. Correspondencia: Yasmine Sotomayor

(yasminesotomayor@gmail.com)

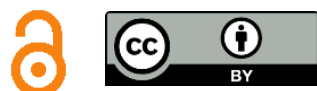

Recibido: 15 de Julio del 2020 Aprobado: 20 de Septiembre del 2020 Publicado: 30 de Septiembre del 2020 


\section{INTRODUCCIÓN}

La Organización Mundial de la Salud define como adolescencia al grupo humano que se encuentra entre 10 y 19 años, considerando "adolescentes tempranos" a quienes presentan entre 10 y 14 años, mientras que entre los 15 y 19 se denominan "adolescentes tardíos". La adolescencia, etapa que atraviesan el $30 \%$ de la población de América Latina y el Caribe, ha de interpretarse como un fenómeno social, cultural e históricamente determinado, siendo el o la adolescente producto y agente en todo proceso recurrente cognoscible de constitución e interpretación de las realidades negociadas con otros. (1) Muchos de los comportamientos que se establecen durante la adolescencia pueden tener efectos persistentes en la edad adulta, por ello se recomienda abordar aspectos tales como la maternidad temprana, las infecciones de transmisión sexual (ITS), el comportamiento relacionado con la búsqueda de atención en salud, la violencia y los comportamientos de riesgo. (2)

El término "educación sexual" describe la educación (proceso de informar, instruir y trasmitir valores) a cerca del sexo en las diferentes etapas del desarrollo, considerando a la sexualidad en su conjunto, con la finalidad de que los individuos que la reciben logren alcanzar una satisfactoria sexualidad en un escenario de libertad y responsabilidad. (3) Actualmente la educación sexual va tomando más temas de importancia, y no netamente las alteraciones en la esfera sexual, considerando temas como la autoestima, el enamoramiento y los valores. (4) Impartir una educación sexual es enseñar sobre sexualidad, donde se deba facilitar a los adolescentes una adecuada interacción para las relaciones interpersonales. (5) Debe buscar también enseñar al individuo a vivir sanamente su sexualidad y goce del placer con el ejercicio de sus derechos en el tema de la sexualidad. La educación sexual es efectiva si se es capaz de legitimar el sexo como un factor en la personalidad y consiga establecer la difícil diagonal entre "lo que es" y "lo que debe ser", ayudando a obtener un espíritu crítico que, a su vez, no cree inadaptados a la sociedad en el que se va desarrollando la vida del niño. (6).

En este sentido, el objetivo de la educación sexual es favorecer la aceptación positiva de la propia identidad sexual, desligada de elementos discriminatorios de género y las vivencias de unas relaciones interpersonales equilibradas y satisfactorias. Son los esfuerzos pedagógicos los que consiguen que la información adquiera importancia. $(7,8)$ La cuestión sería llevar a cabo programas de intervención en educación sexual paralelos a los avances sociales, es imprescindible que los programas de intervención escolares deban de tener un soporte triangular, que hace alusión a una educación que parte primero del padre y la madre, los docentes en los centros educativos y de los profesionales de la salud. (7) Esta educación debe estar contemplada en un largo tiempo, pues no es un conjunto de informaciones o contenidos. (9) Hay quienes resaltan que la educación sexual comienza en el útero materno con el intercambio de estímulos que recibe el feto desde una madre y una sociedad, aquello ama o rechaza a este nuevo ser; al nacer, lo que halla, ve, oye, palpa y cómo lo sostienen, comienzan a fundar los gustos en su futuro. (10) Es básico empezar la educación sexual desde temprano y de ahí ir desarrollándola continua y progresivamente de acuerdo con el grupo etario.

Existen 3 estadios para la educación sexual. El primer estadio es en la niñez donde prima la idea de hablarles siempre con la verdad, sobre todo contestando correctamente las interrogantes que existan. El siguiente estadio es el de los pre púberes y púberes, donde la enseñanza está centrada en la reproducción animal y vegetal, en este estadio es importante la participación de profesionales de salud junto al profesor. En la tercera etapa, comprendida entre los 14 a 17 años, es donde se debe brindar la información completa (5). Hasta ahora los 7 años era la edad mínima considerada para entender el tema de prevención en salud, y a los 11 años el pensamiento operacional formal permite la comprensión de lo que a cada uno le hace bien o mal. Hoy debemos diseñar programas desde el preescolar. (11)

Los estudios descritos hasta el momento han buscado brindar, bajo valores cuantitativos, una visión de la realidad actual con respecto al grado o nivel de conocimiento que presentan los estudiantes o tutores en temas de salud sexual, sin embargo, no se ha ahondado en los motivos o percepciones personales que puedan explicar la problemática con respecto al poco conocimiento sobre este tema. Por ello 
planteamos una investigación que presente el objetivo de Valorar las percepciones de la función orientadora en educación sexual desde la perspectiva del tutor y el alumno en la Institución Educativa Isabel Chimpu Ocllo.

\section{MATERIAL Y MÉTODOS}

Estudio de enfoque cualitativo, con diseño retrospectivo de corte transversal. Este estudio se realizó en la Institución Educativa Isabel Chimpu Ocllo, perteneciente a la UGEL 02 del distrito del Rímac, en Lima. La institución pública mixta cuenta con el nivel secundario de formación regular. El colegio fue elegido para este trabajo de investigación debido a que se ubica en el distrito de San Martín de Porres, un distrito en el cual existen muchos casos de embarazo adolescente y problemas en cuanto a la salud sexual y reproductiva.

Se contó con dos poblaciones de estudio, la primera estuvo conformada por todos los docentes de la institución, los cuales ascienden a un total de 45. La segunda población estuvo conformada por todos los estudiantes del colegio, los cuales ascienden a un total de 660 alumnos matriculados. Para los docentes se consideró como criterio de inclusión el tener experiencia como tutor en cuarto y quinto año de educación secundaria, mientras que para los alumnos se consideró a aquellos estudiantes del quinto grado de educación secundaria y que presentaran menos de 19 años. Se utilizó como tipo de muestra, la muestra dirigida o no probabilística, específicamente aquella de participantes voluntarios. Para determinar el tamaño muestral se siguió el criterio de "saturación", el cual nos facilita operativamente la determinación de este. Finalmente se trabajó con un total de siete tutores (dos varones y cinco mujeres) y diez alumnos (cinco mujeres y varones respectivamente).

Las variables o categorías en las cuales se enmarcaron las preguntas durante la entrevista fueron cuatro: 1) Percepciones sobre la función orientadora en educación sexual; 2) Percepciones sobre la función orientadora en temas trascendentales; 3) Percepciones sobre la función orientadora en temas de controversia de la educación sexual y 4) Percepciones sobre la función orientadora de otros agentes involucrados. Las cuatro categorías fueron consultadas tanto a los tutores como a los alumnos.
Para la recolección de los datos se utilizó la técnica de la entrevista a profundidad, la cual nos permitió obtener una información más completa y detallada de los entrevistados. En esta investigación se empleó como instrumento una guía de entrevista exclusiva para tutores y otra para los alumnos. Dentro de la ejecución se realizó una prueba piloto a fin de realizar los ajustes necesarios a los instrumentos de investigación. El análisis se realizó mediante el modelo de la Teoría Fundamentada, el cual fue realizado por las dos autoras de la presente investigación.

La investigación fue parte de la tesis de grado en obstetricia, por lo cual se contó con la revisión y aprobación de la Escuela Profesional de Obstetricia de la Universidad Nacional Mayor de San Marcos, así como los permisos correspondientes en la institución educativa. Así mismo todos los participantes aceptaron participar en la entrevista y su grabación en audio mediante un consentimiento informado (en caso de los tutores) y asentimiento informado (en caso de los estudiantes), el cual contemplaba la posibilidad de retirarse del estudio si es que lo requiere. Se mantuvo durante todo el estudio un carácter neutro para evitar presiones de carácter moral, conservando así el diálogo auténtico, así como se cuidó la confidencialidad de los participantes.

\section{RESULTADOS}

Los resultados serán abordados en dos segmentos, en primer lugar, las respuestas obtenidas por los tutores y a continuación las encontradas por los estudiantes, a fin de contrastar las percepciones de cada grupo. Las respuestas reportadas en la presente investigación corresponden a aquellas que mostraron ser recurrentes.

Al evaluar percepciones del tutor sobre su función orientadora en educación sexual, se encontró que la hora de tutoría llega a ser el único espacio disponible para atender y orientar a los estudiantes, sin embargo, algunos docentes expresan su descontento por las limitaciones que presentan respecto al tema de educación sexual y el contexto social que presentan los estudiantes, el cual en algunas situaciones es difícil de manejar. Por parte de los estudiantes, ellos llegan a identificar la hora de tutoría como un espacio para orientar, sin embargo, existen quienes desmerecen la actividad. Así mismo, los alumnos buscan en el tutor características como empatía, amistad, sinceridad. 


\begin{tabular}{|c|c|c|}
\hline & Tutores & Alumnos \\
\hline 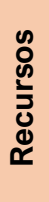 & $\begin{array}{l}\text { "Me parece muy positiva esta oportunidad que } \\
\text { tengo (tiempo de tutoría) porque pienso que se les } \\
\text { está educando, y dejar atrás el tabú, y hacer las } \\
\text { cosas como deben ser..." (Tutora 1) }\end{array}$ & $\begin{array}{l}\text { "Para mí la hora de tutoría está bien que sea una } \\
\text { hora nada más... la mayoría de los profesores } \\
\text { hablan y hablan nada más y no aconsejan y no sé } \\
\text { tratan otros temas y no nos ayudan tampoco sobre } \\
\text { sexualidad..." (Alumna 4) }\end{array}$ \\
\hline 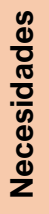 & $\begin{array}{l}\text { "Hablar de educación sexual con los alumnos es } \\
\text { bastante importante, pero... en si yo un poco me } \\
\text { siento corta ... para mí hablar de sexualidad es un } \\
\text { poquito incomodo, bueno a mí se me vienen todos } \\
\text { los colores encima" (Tutora 2) }\end{array}$ & $\begin{array}{l}\text { "Nos gustaría que fuera un tutor casi como nuestro } \\
\text { amigo, que nos hable y que sepa escuchar y que } \\
\text { esté ahí al lado de nosotros y que nos den la } \\
\text { suficiente confianza para poder tratar más con ello" } \\
\text { (Alumno 3) }\end{array}$ \\
\hline 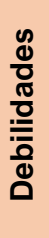 & $\begin{array}{l}\text { "Otra gran debilidad es que nuestra población son } \\
\text { muchachitos o muchachitas que vienen de hogares } \\
\text { disfuncionales que viven con el hermano o viven } \\
\text { con el tío. Entonces quizás esa pueda ser una } \\
\text { puerta por donde ellos quizás puedan hacer cosas } \\
\text { que no deban hacer" (Tutora 3) }\end{array}$ & $\begin{array}{l}\text { "En tutoría, ahí hablamos entre nosotros pe, porque } \\
\text { el tutor no habla mucho sobre el } \\
\text { tema...habla...sobre otras cosas se queda sentado } \\
\text { o se va. A veces no venía tampoco. Nos dejaban o } \\
\text { sea hora libre". (Alumno 1) }\end{array}$ \\
\hline 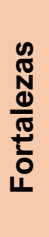 & $\begin{array}{l}\text { "La fortaleza que hay, por lo menos... para mi es } \\
\text { positivo y me siento preparado para hacerlo... y de } \\
\text { los estudiantes ese deseo de querer aprender y } \\
\text { conocer más" (Tutor 1) }\end{array}$ & $\begin{array}{l}\text { "Según mi opinión, cada profesor acá ha tenido sus } \\
\text { años y durante esos años ha debido tener tutoría y } \\
\text { esa experiencia ha ido abarcándose más a más a } \\
\text { cada salón por lo menos que deben tener } \\
\text { experiencia o ya estar un poco preparada". } \\
\text { (Alumno 2) }\end{array}$ \\
\hline
\end{tabular}

Figura 1. Percepciones de los tutores y alumnos sobre la función orientadora en educación sexual.

Por último, se identificaron como fortalezas los años de experiencia que presentan los tutores, aunque existen quienes adoptan actitudes negativas. (Figura 1).

Con respecto a la función que cumplen los tutores en la orientación de temas trascendentales de la educación sexual, se encontró que, al igual que en la dimensión anterior, algunos tutores reconocieron ciertas limitaciones para abordar temas tales como el "plan de vida" o "autoestima", a diferencia del "enamoramiento", donde refirieron presentar mayor facilidad dentro de la consejería. Se halló que los tutores desarrollan con responsabilidad y preocupación su función orientadora respecto al inicio de las relaciones sexuales, revelándose un escaso apoyo por parte de la familia del estudiante. Con respecto al embarazo y los temas referidos a las infecciones de trasmisión sexual, los tutores coinciden en que debe ser de consejería constante. En el caso de los alumnos, ellos consideran que la consejería sobre el plan de vida y autoestima se realizó con la importancia debida, aunque refieren que debe tratarse de manera más profunda, así mismo perciben que la consejería sobre el embarazo es poco especializada. (Figura 2)

Se evaluó luego la función orientadora en temas controversiales, considerando entre ellos al aborto, los métodos anticonceptivos y la homosexualidad. Con respecto al aborto y la homosexualidad se encontró que los factores socioculturales pueden influenciar en la información brindada, así como también el uso de recursos audiovisuales fue muy utilizado en este tema. Dentro de la consejería sobre métodos anticonceptivos, se observó que a pesar de la carencia de información especializada existe apertura a tratar el tema. Los alumnos por su parte consideraron los temas tratados como importantes, sin embargo, solicitaban que se amplíe la información al respecto. Destaca que los alumnos percibieron resistencia o poco interés por parte de los tutores para hablar sobre la homosexualidad en los horarios de tutoría. (Figura 3) 
revistamaternofetal.com

\section{Tutores}

"Como que allí me faltó, yo soy consciente que me faltó... o sea yo aplicaba las dinámicas que nos dan las guías, los manuales; pero como que sí, me faltó" (Tutora 1)

"El autoestima en general es un tema muy importante, es bastante amplio, pero casi siempre se programan muy pocas clases y... no están tomando la conciencia del tema a tratar, lo toman al juego, no lo comprenden, como que lo escucharon y luego se les olvidó" (Tutora 3)

올

"La importancia es que ellos sepan afrontar la edad que tienen, que están en una edad de ilusión que siente atracción por el sexo opuesto, la importancia sería orientarlos de cómo tomarlo ese enamoramiento. Así que por alli hay que trabajar bastante porque si es un tema crucial" (Tutor 2)

"De nada sirve si tú le dices al alumno "ten tu primera relación sexual cuando realmente sientas que es el amor de tu vida" ... porque de repente en su casa viven en una promiscuidad terrible, cuando ven que la mamá trabaja de esa manera, es una realidad que tenemos acá, no viven en valores, entonces lo que uno les pueda decir, para ellos, es abstracto" (Tutora 1)

"De hecho no estoy de acuerdo con eso por ello es que tratamos en la mayoría de lo posible de transmitirle al adolescente "el no salir embarazada tan rápidamente", ya es un problema social para mí" (Tutor 1)

"Me parece que es un tema bastante importante para ellos, es de gran preocupación a nivel mundial, porque hay enfermedades que ya no tienen cura no, como por ejemplo el VIH" (Tutora 3)
Alumnos

"El tema plan de vida me pareció muy bueno... nos dice que es lo que queremos ser más adelante o sea saliendo del colegio que vas a estudiar, nuestro objetivo, cómo lo íbamos a plantear y cómo lo ibamos a desarrollar..." (Alumna 1)

"La clase de autoestima sí...un poco aburrida, el mismo concepto, era casi ya repetitivo a los años pasados, pero en realidad la persona sí debería estudiar esa parte" (Alumno 4)

"Sí se sintió normal sí, interactuamos y sí la sentí bien cómoda... ya los chistes que nosotras hacíamos como que se reía y como que se ponía a nuestro nivel y sí fue chévere esa clase, me gustó, sí me gustó". (Alumna 3)

"El tutor nos comenzaba a orientar (sobre inicio de relaciones sexuales),...es muy muy íntimo de cada uno, y no es fácil hablar,... en parte a veces renegaba porque algunos se reían, lo tomaban a la broma, porque lo ven a las relaciones como algo extraño, pero en parte ya tenemos la edad suficiente como para poder conocer" (Alumna 1)

"El embarazo es un tema que teníamos muchas dudas, ... sí nos explicó, e incluso nos trajo folletos de embarazo en la adolescencia, embarazo precoz y nos hizo conocer más del tema. Sí estuvo preparado porque nos supo orientar lo principal...pero me hubiera gustado conocer algo más especializado, más fundamentado". (Alumna 1)

"Se sintió serio porque el VIH causa muerte y... como que malogra la vida de una persona. Si estuvo preparado, cómodo y seguro". (Alumno 1)

ITS: Infecciones de transmisión sexual

Figura 2. Percepciones de los tutores y alumnos sobre la función orientadora en temas trascendentales de la educación sexual. 
"Usted sabe que las personas somos parte afectiva, parte sentimental... a veces nosotros les mostramos videos de cómo es un aborto provocado y las niñas sales muy sensibilizadas, los videos nos ayudan bastante porque ellas preguntan "¿Qué señorita así es?" sí les digo ahí están viéndolo... Pero también hay que saber manejarlo ..." (Tutora 5)

"iMétodos anticonceptivos como ingesta, ampollas, pastillas, los DIU, o los anovulatorios, no estoy de acuerdo, pero con el preservativo sí! Definitivamente en eso sí estoy de acuerdo porque no les afecta ni al uno ni al otro" (Tutora 3)

"Bueno tocar la homosexualidad no fue de mucho agrado para mí, hay que hablarle a los chicos de esto, porque estamos viviendo en un mundo con este tipo de sexualidad, para que ellos también sepan diferenciar y puedan ver que Dios solo ha creado dos sexos, yo les pongo ejemplo con los animalitos, que el animalito es macho o es hembra" (Tutora 2)
"La tutora, nos dio a entender que el aborto, puede ser algo triste y no solamente destruye a la madre que lo piensa abortar, sino también destruye a una criatura que no conoce la vida, es un tema de mucha tristeza si lo notas y más un video que yo he visto por mi parte, es muy traumático" (Alumno 2)

"Sí... sí se podría decir, un poco (conforme con la información recibida). Porque ya sé que cuando tenga relaciones sexuales debo usar condón, preservativo, y cuando ya deba y quiera tener un hijo o ser profesional, ahi sí, al natural, jejeje (risas)". (Alumno 1)

" $\mathrm{mmm}$ homosexualidad no nos han hablado, creo que no (sonrisa). Me hubiera gustado porque no sé realmente que significa... y creo que es importante que nos hablen...porque en otros países creo que ya es común ¿no? Tener el tercer sexo ...y tengo ciertas dudas, ...como que porque se vuelven asi" (Alumna 2)

Figura 3. Percepciones de los tutores y alumnos sobre la función orientadora en temas controversiales de la educación sexual.

\begin{tabular}{|c|c|c|}
\hline & Tutores & Alumnos \\
\hline 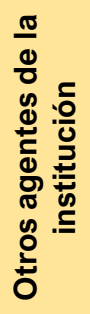 & $\begin{array}{l}\text { "Que la señorita psicóloga está al tanto de los } \\
\text { alumnos, cualquier caso está allí para solucionar } \\
\text { cualquier caso que el tutor no pueda solucionar, si } \\
\text { en caso se necesita de personas más avezadas" } \\
\text { (Tutor 2) }\end{array}$ & $\begin{array}{l}\text { "Los tutores deberían ayudarnos más como el } \\
\text { auxiliar que le tengo confianza porque la conozco } \\
\text { desde primero, o la profesora de sociales que nos } \\
\text { habla directo o como el profesor de Persona y } \\
\text { familia. El profesor de Persona y familia tiene más } \\
\text { edad y tiene más experiencia sobre esto, o sea, él } \\
\text { nos habla normal, abiertamente, lo dice todo, sin } \\
\text { ajos, lo dice normal ...." (Alumna 2) }\end{array}$ \\
\hline 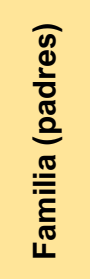 & $\begin{array}{l}\text { "En este colegio se evidencia muchos padres con } \\
\text { demasiadas horas de trabajo y los chicos se quedan } \\
\text { solos, no hay orientación, no hay persona que les } \\
\text { guie, no hay de alguna manera como se puede } \\
\text { decir, represión o respeto a una persona mayor... y } \\
\text { ellos están desinformados... entonces se da la } \\
\text { relación sexual a muy temprana edad" (Tutora 3) }\end{array}$ & $\begin{array}{l}\text { "Ellos como papás deben informarnos porque a } \\
\text { veces cometemos errores y no sabemos. Yo creo } \\
\text { que esa educación se debe hacer en el colegio, pero } \\
\text { tanto en el colegio como en la casa" (Alumna 4) }\end{array}$ \\
\hline 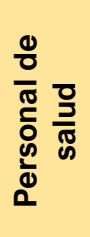 & $\begin{array}{l}\text { "Les digo a ellos plenamente que pregunten, } \\
\text { pueden estar con dudas pregúntenlo a cualquiera } \\
\text { de los profesionales, pero que no se queden con la } \\
\text { influencia de la calle... porque eso es negativo, } \\
\text { tremendamente" (Tutor 1) }\end{array}$ & $\begin{array}{l}\text { "Vienen por temporadas (los del MINSA) a } \\
\text { hablarnos de varios temas las veces que vienen } \\
\text { somos muy bien aconsejados y orientados por ellos, } \\
\text { cubren nuestras expectativas, nos dan folletos, nos } \\
\text { enseñan videos, nos dan indicaciones y sí hablan } \\
\text { muy ampliamente del tema" (Alumna 1) }\end{array}$ \\
\hline
\end{tabular}

Figura 4. Percepciones de los tutores y alumnos sobre la función orientadora de los agentes que intervienen en la educación sexual 
Por último, en la Figura 4, se analizaron las percepciones con respecto a los agentes que intervienen en la educación sexual. Por parte de los tutores se identificó al personal de psicología como un apoyo complementario en la orientación, tal como también se consideró al profesional de salud externo o estudiantes de diversas universidades que acudían al colegio para brindar charlas informativas. Así mismo se evidenció que el contexto familiar del alumno contribuye a la mala toma de decisiones con respecto a su vida sexual. Con respecto a los alumnos, ellos refirieron que dentro de la institución el tutor y la psicóloga tienen poca actividad, por lo cual su labor está desvalorizada, por el contrario, los estudiantes resaltan el apoyo de otros agentes. Así mismo, al consultarles sobre la labor del personal de salud ellos refieren que sintieron que la información brindada fue completa y especializada.

\section{DISCUSIÓN}

El objetivo de nuestra investigación fue valorar las percepciones de la función orientadora en educación sexual desde la perspectiva del tutor y el alumno. Al respecto, insistimos en que no se encontraron investigaciones previas que busquen nuestros mismos objetivos; sin embargo, se tomaron en cuenta aquellas que sí guardan relación con nuestro estudio.

Respecto a las deficiencias institucionales, tanto alumnos como tutores expresaron insatisfacción por el corto tiempo que se destina para la tutoría, considerando que se cuenta con una hora pedagógica a la semana, tiempo que resulta mínimo para la educación sexual. Así mismo, alumnos y tutores perciben que el otro factor que afecta a la función orientadora en educación sexual es la necesidad de capacitaciones especializadas dirigidas a los tutores, pues con ellas se lograría que los docentes obtengan un óptimo nivel de información y metodología para abordar estos temas. (12) En este aspecto, los tutores y alumnos refirieron dos características importantes que pueden dar respuesta a su deficiencia.

Como primera característica relevante se expresaron las pocas o inexistentes capacitaciones en tutoría, en este punto mencionaron que en las pocas capacitaciones recibidas no abordan temas específicos en educación sexual, sumado a esta desventaja precisaron que a dichas capacitaciones solo asiste un número limitado de tutores, a quienes se les asigna replicar la información recibida con sus pares. Este hecho condiciona que no todos los tutores adquieran un adecuado y uniforme nivel de capacitación, teniendo en cuenta que la capacitación comprende no solo el aspecto cognitivo sino también el metodológico. (13) En este punto la investigación desarrollada por Testa $\mathrm{M}$ et al, pone en evidencia la necesidad de capacitar en el área sexual a los docentes, pues entre los resultados que obtuvieron el manejo de información y terminología correcta relacionada con temas en el área sexual tuvo una tendencia baja, con presencia de un $40 \%$ de errores de concepto; observándose, además, un porcentaje considerable con bajo nivel cognitivo sexual en los docentes estudiados. Asimismo, respecto a las estrategias didácticas, los docentes muestran un bajo nivel en la utilización y dominio de recursos y estrategias que deben utilizarse para impartir exitosamente educación sexual. (14) Al respecto, Rivera $\mathrm{R}$ et al demuestran que el docente no cuenta con los conocimientos necesarios para responder de manera adecuada las principales dudas o preguntas que tienen los estudiantes respecto a la sexualidad, y aun cuando presentan una actitud positiva, ésta puede ser dudosa en la medida en que el profesor manifiesta ignorancia en el conocimiento científico de la sexualidad. (15)

Como segunda característica se halló que todos los docentes, independientemente de su especialidad, pueden ser designados como tutores. Esto supone una desventaja, pues no todos tienen el adecuado nivel de conocimiento y manejo en temas de educación sexual, tal como se demuestra en nuestros resultados, donde los tutores perciben que existe cierta facilidad y dominio para el manejo de temas en educación sexual por parte de aquellos docentes que imparten materias de ciencias o afines a las relaciones humanas. Ahora bien, respecto a la dimensión profesional y personal, el estudio muestra que la disposición y la actitud con la que desarrollan su función de tutoría estaría influenciada por los valores, creencias e idiosincrasia propias de cada docente, ya que muchas veces los temas a tratar entran en conflicto con estos.

En ocasiones la función orientadora en educación sexual se complementa con el trabajo multisectorial de profesionales y estudiantes de salud. Respecto a este punto los tutores opinan que las intervenciones realizadas por estos agentes son poco innovadoras, 
que sus alumnos necesitan otras estrategias para lograr los objetivos. Esta opinión es compartida por los alumnos, sin embargo, a pesar de estas deficiencias, los alumnos resaltan la información completa y especializada que reciben de estos agentes de salud. Por lo tanto, se ve la necesidad de que exista un consenso entre ambos sectores para abordar una educación sexual integral, especializada y coordinada. (16) Esta última observación se refleja en la investigación realizada por Marcelo $A$, donde se encontró que para el sector salud y los profesionales médicos la concepción y el enfoque que le dan a la educación en sexualidad es un enfoque de riesgo que se centra en la sexualidad como causante de embarazo o enfermedades de transmisión sexual; mientras que el sector educación le da un enfoque más biologista, lo que conllevaría a que esta educación sexual no se está desarrollando de manera adecuada. (17)

Es necesario considerar que los resultados hallados en la presente investigación solo podrían ser transferibles a aquellas instituciones educativas que presenten características similares, que en este caso presentó una alta proporción de embarazos adolescentes en el distrito y en dicha institución. Se recomienda además que el profesional obstetra se capacite, dentro de su formación universitaria, en competencias referentes al área educativa en temas relacionados a la orientación y consejería en educación sexual para adolescentes.

Se concluye que la función orientadora en educación sexual es una responsabilidad importante hacia los alumnos, que depende del compromiso y la actitud de cada tutor, pero que se ve limitada por el poco tiempo asignado y por la carencia de información y capacitación (hacia los docentes) en temas de educación sexual. Los temas de enamoramiento, autoestima y plan de vida son más manejables, en comparación al inicio de relaciones sexuales, embarazo e infecciones de transmisión sexual. Así mismo, los temas como aborto, métodos anticonceptivos y homosexualidad son difíciles de abordar para los tutores.

Se requiere de una Educación Sexual Integral con enfoques emergentes como los de enfoque de género, de derechos e inclusión y atención a la diversidad sexual; pero que, además, incluya a otros agentes formadores como los padres de familia $y$ personal de salud capacitado en salud sexual y reproductiva. Estas intervenciones deben ser innovadoras y con una metodología adecuada y amigable, para poder involucrar activamente a los y las adolescentes. 


\section{REFERENCIAS}

1. Chávez S. Concepciones y percepciones sobre los derechos sexuales y reproductivos en la adolescencia. Centro de Promoción y Defensa de los Derechos Sexuales y Reproductivos, PROMSEX. Lima, Perú. 2007. [Link]

2. Organización Panamericana de la Salud. La salud sexual y reproductiva del joven y el adolescente: Oportunidades, enfoques y opciones. 2008. [Link]

3. Farré J. Las edades del sexo. Enciclopedia de la sexualidad: Volumen 4: 63 - 65. Barcelona, España: Grupo Océano Editorial. 2003.

4. Ministerio de Educación. Lineamientos para una educación sexual integral. Dirección de Tutoría y Orientación Educativa, Documento de trabajo. Lima, Perú. Abril, 2008.

5. Farré J, Flora F. ¿Educar el sexo o educar para el sexo? Enciclopedia de la sexualidad: Volumen 4: 594 - 616. Barcelona, España: Grupo Océano Editorial. 2003.

6. Ávila A. ¿Educar es prevenir? Enciclopedia de la sexualidad: Volumen
4: 606 - 607. Barcelona, España: Grupo Océano Editorial.2003.

7. Donini F, García A, Villa A. Mitos y falacias sexuales existentes en los agentes educativos (Familia y escuela). Buenos Aires, Argentina: Ediciones novedades educativas. Junio, 2005.

8. Talburt S, Staindberg S. Educación precoz: Sexualidad e información. Pensando querer sexualidad, cultura y educación. España. 2005.

9. Altable C. Educación sentimental y erótica para adolescentes: Educación sexual experimental. Madrid, España. Miño y Dávila editores; 2000.

10. Guelmán de Jaukín M. Infancia y adolescencia entre necios y sabios. Rosario, Argentina. Homo Sapiens Ediciones; 2005.

11. López F. Mi hijo(a) adolescente: La educación sexual de los hijos. Madrid, España: Pirámide, Grupo Ancaya. 2005.

12. Bautista-Hernández LA. Conocimientos, actitudes y opiniones de las y los universitarios oaxaqueños respecto a su sexualidad y la importancia de la educación sexual en el nivel de educación superior. Centr Regi Inves Psicol. 2008; 2 (1): 71 - 84.

13. Romero de Castilla G. Lora R. Cañete E. Adolescentes y fuentes de información de sexualidad: preferencias y utilidad percibida. Atención primaria. 2001; 27(1). [Link]

14. Mirian Testa, Maria Estela Nuñez, Fredy Ruiz, Alexia Senior. Nivel de educación sexual de los docentes. Venezuela. 2002.

15. Ramírez C. Conocimientos y actitudes hacia la sexualidad en profesores de educación secundaria.

16. Ramiro M. Percepciones de profesores portugueses sobre la educación sexual. Rev Panam Salud Publica. 2008; 42 (4).

17. Marcelo A. Sexualidad y reproducción en el escenario educativo: discurso y prácticas de adultos del sector educación y salud en escuelas medias de la ciudad de buenos Aires. Buenos Aires, Argentina. 2005. 